\title{
Comments on the Basis of Wave Mechanics
}

\author{
Mohamed Ashraf Farouk* \\ Department of Physics, Egypt
}

\section{ISSN: 2576-8840}

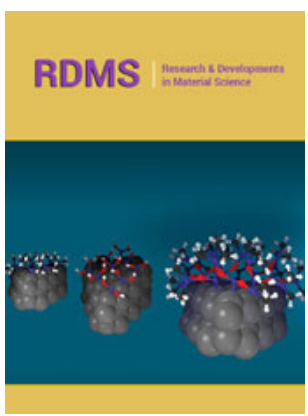

*Corresponding author: Mohamed Ashraf Farouk, Department of Physics, Ain Shams University, Arab Republic of Egypt, Egypt

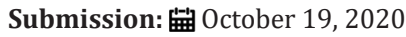

Published: November 10, 2020

Volume 14 - Issue 2

How to cite this article: Mohamed Ashraf Farouk. Mohamed Ashraf Farouk. Res Dev Material Sci. 14(2). RDMS.000835. 2020. DOI: 10.31031/RDMS.2020.14.000835

Copyright@ Mohamed Ashraf Farouk. This article is distributed under the terms of the Creative Commons Attribution 4.0 International License, which permits unrestricted use and redistribution provided that the original author and source are credited.

\section{Abstract}

Showing that the origin of the derivation of the fundamental equation of wave mechanics, the Schrödinger's equation is absolutely baseless.

\section{Introduction}

Copenhagen physicists said; The electronics that we use today-radio, televisions, computers, lasers, MRI machines GPS-communications- depend on calculations using Schrodinger's equation plus a lot of other Mathematics and Measurements, our idea of "shut up and calculate" has worked very well for us technologically. My comment's these technological achievements do not justify that the equation itself as (I will try show) has any true scientific basis. In 1925, Erwin Schrodinger wrote excitingly to Einstein. "I have been intensely concerned these days with Louis de Broglie's theory [1]. It is extraordinary, exciting, but still has some very grave difficulties". So regardless of you are consider Schrodinger's equation is a fundamental equation (has no derivation from simples forms) or derived one, you cannot deny first that Schrodinger's equation is a wave equation and second it is shows how (de Broglie hypothesis of) matter wave change with time. i.e. Schrodinger equation describe the evaluation of the physical system in which the quantum effects, the "Waveparticle" duality are significant.

\section{About the derivation}

There are various ways for the derivations of Schrödinger's equation in physics textbooks, I will try to summarize it; most of the physicists thinking was as the following:

A. To describe a wave equation for a particle such as an electron, we expect that its wave function will satisfy a differential equation that is akin to (but not identical) to the classical wave equation.

B. So, physicists starting their derivation with writing an expression for the wave function in term of its wavelength and frequency.

C. This expression of the wave function is convenient since we already know that the wavelength " $\lambda$ " and the frequency " $v$ " can be written in terms of the linear momentum " $\rho$ " and the total energy "E" of the particle being described; $\lambda=h / \rho, E=h \nu$.

D. Then the physicists partially differentiate the wave function " $\psi$ " with respect to spatial coordinate twice, and partially differentiate " $\psi$ " with respect to time once and deducing both the momentum operator and the total energy operator [2].

E. Then the physicists set up the Hamiltonian equation similar to classical mechanics.

$$
\mathrm{H}=\mathrm{T}+v \quad \mathrm{E}=\rho^{2} / 2 \mathrm{~m}+v
$$

F. Then they convert each momentum component when it appears into an operator. Similarly, they transform the total energy component into an energy operator.

G. Now they got the wave function which would be a function of all the coordinates of the system and of the time.

$$
\mathrm{H} \psi=\mathrm{ih} \mathrm{d} \psi / \mathrm{dt}
$$


H. The Hamiltonian is a function used to express the energy of the system in terms of its momentum and the positional coordinates, i.e. in Hamiltonian equation; the usual equations used in Mechanics (based on forces) are replaced by equations expressed in terms of momentum).

I. Physicists said that; Schrodinger's equation In the variable $\psi$ is the fundamental equation of quantum mechanics in the same sense that the second law of motion is the fundamental equation of Newtonian Mechanics.

J. Physicists showed how Schrodinger's equation could be formulated for any mechanical problem whatever, physicists said; the validity of any fundamental mathematical equation reside on its agreement with experiment. They have no doubt that the formulation of Schrodinger's equation provides the fundamental and the correct basis for the quantum theory.

K. Finally, physicists showed how to set up Schrodinger's equation for many electrons problems. "The mathematics become extremely complicated, so hard that we are still working on methods of solving it" [3].

\section{My comments}

A. i.e. The physicists (Now) today are busy with the mathematical details of the solution of Schrodinger's equation, and No one notice that may be all these complicated details might Hide an important fact which is that the original Mathematical formulation itself, the Schrodinger's equation itself may be absolutely baseless.

B. I can conclude that the common factor among all of these different ways of deriving Schrodinger's equation is the substituting about the wavelength and the frequency (of the matter wave) with the momentum and Energy of the particle $\lambda=h / \rho, v=E / h$ without such Einstein and de Broglie relations, the constant $\mathrm{h}$ will never appear in Schrodinger's equation. So, let us analyze the origin of de Broglie-Einstein Mathematical formula [4]. Because I think that the origin mistake lies in the fact that Schrodinger took seriously the suggestion of de Broglie, which is that there might be a wave accompanying the motion of the particle. Then "Schrodinger's" thought that if this were so, then one could guess the form of the wave equation of the resulting wave motion". Let us analyze de Broglie's work.

C. The central concept from which de Broglie view is originated is "The Symmetrical of Nature", That his thinking was as follows; since our observable universe is composed entirely of light and Matter, and since light which is waves (as he think) has particle Nature, thus we can conclude that matter which is composed of particles may have a wave-like Nature", i.e. he suppose the Analogy between light and matter, he create the equation $\lambda=h / \rho$.

D. de Broglie wrote that the energy and the momentum of the photon is suggestive of the intimate connection between the quantum and wave theories

$$
\mathrm{E}=\mathrm{h} v \quad \rho=\mathrm{h} / \lambda
$$

E. de Broglie thought that these two formula Bridge the gap between the world of mechanics in which the energy and the momentum reign and the world of waves described by $v, \lambda$ by other words believed that the two different and separated aspects are interlinked by these two relations [5].

\section{My comments}

I considered that any trial to merging or connecting the wave and the particle theories mathematically must be failed (Figure 1). I would like to say that the ideas of entities which can show the properties of both particles and waves, or of particles guided by waves associated with them, or of waves which carry energy in discrete particle units, are all rather impossible to grasp, if presented on their own or embodied in mathematical equations. For example: "In his book titled "Light and vacuum" Constantin Meis wrote; "Today, we perfectly understand the behavior Laws of light but our Mathematical representation of its "Wave-particle" nature and its relation-ship to the vacuum is still incomplete". I clarified in my previous essays [1] that light hasn't a wave Nature, I clarified that Neither the double-slit pattern is an interference wave pattern, nor, the single slit pattern is a diffraction wave pattern, also I clarified that light hasn't a particle Nature, I showed that neither the photo-electric effect nor the Compton effect demonstrate the particle nature of light, simply because "there are no photons" and I supposed that not one physicist through the whole world could give us "any pictorial description could help us to understand the Einstein and de Broglie two mathematical relations, simply because the energy of a point particle couldn't depend on frequency [5].

De Broglie made great mistake by extending the false wave particle duality view from the science of optics to mechanics and concerning what is called wave nature of material particles (Davisson and Garner experiment): I would like to say that such experiment could be interpreted as ordinary classical electron scattering not as modern electron's wave diffraction [6]. Finally, to those physicists whom promote that Schrodinger's equation in fundamental equation and has no derivation, I tell them you afraid considering its derived equation because you are terrified that if someone will successes discovering that just one of the steps derivation has no basis neither from logic nor from the real physical world then they know that in such case the whole derivation will collapse, anyway, the equation did not fall from the sky on Schrodinger's mind.

Let's Now Suppose that the Double Slit Pattern is an Interference Wave Pattern

\section{Delayed-choice experiment "strangeness of the quantum world"}

The author of that article said, The experimental results reveal more than ever that; we live in a strange "quantum world" that defies comfortable common-sense interpretation. 


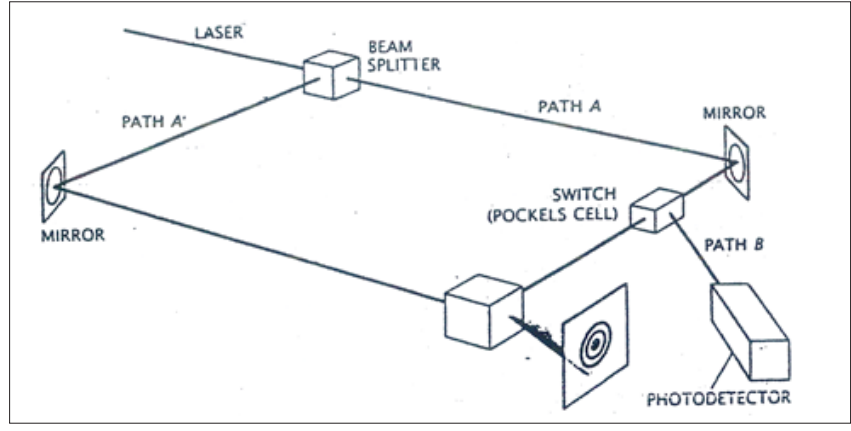

Figure 1:

Delayed-choice experiment: Delayed-choice experiment is another test that reveals the strangeness of the quantum world. A photon impinges on a beam splitter. Two questions about the photon can be asked. Does the photon take a definite route so that it is either transmitted or reflected by the beam splitter, thereby exhibiting a particle like property? Or is the photon in some sense both transmitted and reflected so that it interferes with itself, exhibiting a wavelike property? To find out, a switch is positioned in one of the two paths the photon can take after interacting with the beam splitter (here, path A). If the switch is on, the light is deflected into a photodetector (path B), thereby answering the question of which route and confirming the photon's particle like properties [7]. If the switch is off, the photon is free to interfere with itself (paths $A$ and $A^{\prime}$ ) and produce an interference pattern, demonstrating the photon's wavelike properties. Results from the experiment show that a photon behaves like a wave when wavelike properties are measured and behaves like a particle when particle like properties are measured. Remarkably, the switch was triggered after the photon had interacted with the beam splitter, so that the photon could not have been "informed" whether to behave like a particle and take a definite route or to behave like a wave and propagate simuitaiieouslyalong two routes. This experiment made in Princeton University in 1978 and published in Scientific American Magazine, January 1979 [8].

\section{About bohr's view}

A. Copenhagen: Quantum physicists said that the "waveparticle duality nature is the key feature of quantum mechanics.

B. Earlier bohr: Bohr consider that sometimes there is a real wave but no particles, and some other times there are a particle but no waves. He wrote that a single model is not fully adequate to describe neither light nor matter, the two models are not contradictory but complement each other.

\section{Some physicists understand Bohr view as the following:}

They think that Bohr means that the quantum entities (the fundamental unit of light and matter) is protiform (has a changeable form) such that it appears sometimes as a wave and some other times as a particle. For example; the photon (The fundamental unit of light (the quantum of light) can act as a particle one moment, following a well-defined path like a tiny projectile, and a wave next, overlapping with its ilk produce interference pattern, much like a ripple on the water.

New experiments demonstrate that photons not only switch from wave to particle and back again but can actually herbor both wave and particle at the same time. As written from:

1) Quantum entities: "National center of scientific research in Paris-University of Nice Sophia Antipolis.

2) University of Bristol in England".

However, Bohr view of light is not readily accepted the main reason for this is the apparently contradictory aspects of the two natures; It is difficult to accept the conflicting behavior, that part of time the fundamental unit of light is wave (i.e. a wave is spreads out and occupies a relatively large region of space) and the other part of time the fundamental unit of light is a particle (a particle occupy a definite position in space, it must be very small). Some other physicists said that "the resultant light nature we got depends on the used apparatus; if we set up an apparatus to measure waves, we will find waves. If we set up an apparatus to measure particles we will find particles". However, the physicists cannot readily accept such view also simply because physicists know that the job of apparatus is detection the reality not creating it.

Later Bohr; argued that; "a space-time" description of actual atoms and electrons is not possible. Yet a description of experiments on atoms or electrons must be given in terms of space and time and other concepts familiar to ordinary experience, such as waves and particles [9-12]. We must not let our description of experiments leads us into believing that atoms or electrons themselves actually exist in space and time as particles. The distinction between our interpretations of experiments and what is really happening in Nature is Crucial, to proving that Bohr's opinion is right, the physicists witnessed philosopher Kant. The German philosopher "Immanuel KANT" wrote in his:

\section{Critique of pure reason}

1. "What may be the nature of objects considered as things in themselves [...] is quite unknown to us. We know nothing more than our own mode of perceiving them, which is peculiar to us".

\section{My comments}

1. Right words and wrong implications, they tried to confiscate any future trial to understand what is going on, Bohr's view is originated from invalid inductions and in valid deduction for light phenomenon.

2. It should be noted that I have tried before to introduce a new light model. "The wavy-ray" Model [1]

\section{About max born view}

Max Born said that the particle has an infinite number of possible positions prior to measurements through the wave packet, i.e. the microscopic particle is potentially presence (state) (Mysteriously omnipresent) through the entire wave, the microparticle is sharp localized only at the instant of observation. 


\section{My comments}

There is a great different between saying we do not know the exact position of the particle and saying it hasn't a definite position. Max Born view does not eliminate the simple fact that "The particle can't exist in two points at the same time (simultaneously) which means that it is exist at a particular but unknown point with respect to us which means that Max Born probability wave is due to our ignorance the exact position of the particle which imply that the uncertainty is absolutely not inherent in the nature of a moving microparticle as the physicist thought, beside it is insane to think that the probability could propagate following a regular manner (wave form) [13-15].

\section{Philosophical comment}

Physicist said: Quantum mechanics reflects a spectacular feature of scientific progress. In the critical moments of the history of science. New concepts are not deduced logically from the already familiar knowledge; a logical jump takes place, a new way of thinking and the capability of digesting new notions, No matter how unbelievable, they may seen, are created. This happened in quantum mechanics in the following way; First we had the Mathematical scheme, (second) and then, of course, we had to try to use a reasonable language in connection with it. Finally, we could ask; What concepts does this Mathematical scheme imply, and how do we have to describe nature? The decisive step is always a rather discontinuous step. You can never hope to go by small steps nearer and nearer to the real theory, at one point you are jump, you must really leave the old concepts and try something new, but in any case you can't keep the old concepts. The trouble is that the human mind is accustomed to function within the framework of a definite scheme and switching to another scheme proves to be a very painful operation. "Particles quantum waves ya. A. Smorodinsky Mir Publishers. Moscow”.

\section{My comments}

I found my self forced to remind the reader with the following words.

\section{David hume}

When a philosopher comes up with something that looks like a paradox and is contrary to basic beliefs of ordinary folk [16-19]. It often fares better than it deserves, for two reasons. It is greedily embraced by philosophers. Who think it shows the superiority of their discipline that could discover opinions so far from common beliefs? When something surprising and dazzling confronts us, it gives our minds a pleasurable sort of satisfaction that we can't think is absolutely baseless. These dispositions in philosophers and their disciples give rise to a relation of mutual comfort between them: the former furnish many strange and uncountable opinions, and the latter readily believe them.

\section{Hume: a treatise of human nature. Book I, part II, section} I.

For example: I would like to say it is crazy to the think that the wave function could be considered as an elementary image as the instead image of material point moving along trajectory. In my view the physicists are confused about what it means by the wave aspect of a particle, even de Broglie himself whom ask himself is this wave accompanied the motion of a moving particle, is it of mathematical nature as Max Born said or it is real (actual) wave as Bohr said; de Broglie try to answer the question; is such wave subjective or objective wave? He wrote, the wave which produce diffraction and interference, and affect the motion of the electron must be Real, However the reduction of such wave implies that it is not real. Finally, he wrote, the Real or unreal nature of matter wave remains very obscure. It is so because it is so "current interpretation of wave mechanics page 34" By Louis de Broglie. i.e. In his mind the Riddle remains unresolved. In my view his matter waves cannot be neither subjective nor objective.

\section{Analysis of the Delayed-Choice Experiment}

In my view the results of this experiment is in complete conflict with the quantum theory for the following reasons.

A. The quantum theory does never said that a single photon could behave as a wave because the wave aspect according to the quantum theory only (via the square of wave amplitude) determine the position probability per unit volume that the particle regards as a corpuscle at a given point in space.

B. If the photon energy is split through the two routes this will imply the following:

1. The division of the photon is in conflict with its definition (emitted, absorbed or propagated only as a whole), it cannot be divided, i.e. conflict with the individuality of the photon.

2. If the photon energy is divided, according to the quantum theory, it will means that the division of $v$, say $1 / 2 v$ will means doubling $\lambda$, which means that the light should change its colour after passing the beam splitter which is never happen in laboratory.

3. The complete Interference wave pattern of a single photon is an experimental proof that Max Born view is not true it is an illusion, which tell us that the result should be a spot or the screen.

4. In my view, this is an experimental proof that the photon is not the fundamental unit of light because the division of the photon through both routes without changing its colour which means directly that light consists of entities with an energy less than the photon energy but with the same frequency. In my view the photodetector signal depend only on the rate of b's (how many b's arrived per second), this is the matter and not the receiving of the whole $(\mathrm{hv})$ please see my wavy-ray model interpretations of the photo electric effect.

Again, I would like to say; If the photon divide through both routes and interfere with itself the results should not be a complete interference pattern, because the interference pattern according to quantum theory is formed by commutative arrival of a large number of photons, each one hit the screen at a single point. i.e. according to quantum theory the result should be a spot on the screen, a single photon could never produce a complete in pattern. 
Finally, I would like to say that the author does not understand the quantum theory, he has only one goal to frighten us from trying to use logic to understand the physical world and they are ready to do the impossible to spread abroad falsely the duality idea. Any way this experiment cannot be understood from the photonic point of view.

\section{My view}

To escape from all of these non-sense ideas, Copenhagen physicists spread abroad falsely that Schrodinger's equation is only a TRICK for calculating what we will detect when measuring a microparticles. They said nevertheless, there is no need to prove the equation as it works without understanding what the equation really describes in physical reality. Physicists have been hugely successful using Schrodinger's equation in their calculation [2022]. I can see that we are doing tricks and Manovers of using what we call "representing the observables by quantum mechanics operators" in order to match between the body of our mathematical formalism and the practical experimental results. Yes, we made clowny mathematical tricks, we create many postulates, Hundreds of rules and what we call the approximation techniques in order to narrowing the gap between theoretical mathematical calculation and the experimental results. Physics should never be reduced to be just a trick.

It should be noted that: Very important progress can be made, and has been made since 1926, along these lines, without having to go far in the direction of Numerical solutions of Schrodinger's equation. However, as Prof. Pang Xiao Feng "University of Electronic Science and Technology of China". $5^{\text {th }}$ international conference on wrote in his paper to "Quantum physics And Nuclear Technology" and $6^{\text {th }}$ international conference on "Atomic Physics and Nuclear Physics" November 2019. Rome Italy". He wrote "Our investigations showed that quantum Mechanics have a lot of difficulties and contradictions, which were exhibited and elucidated in details by us through the comparison between the experimental facts and the theoretical results obtained from the solutions of Schrodinger's equation with different potentials. The quantum mechanics can only give the wave features of microscopic particles, cannot always exhibit their corpuscle features. Quantum Mechanics cannot be used to describe correctly and completely the duality of wave and corpuscle features of microscopic particles".

\section{Conclusion}

The basic fundamental equation of wave mechanics, the Schrodinger's equation is absolutely Baseless, we should override it. Physics should get back to speak classically. In my opinion it is just a question of time [23-25].

\section{Open challenge}

Prof. Per-Olav-Lowdin said that; there are three fundamental equivalent theories, "Schrodinger's wave mechanics, and Heisenberg Matrix Mechanics and Dirac q-theory," physicists said that these three theories nearly produce identical numbers for solving the physical atomic problems. I challenge any physicists to give us a Numerical example for any physical problem with its solution by the three different theories. I would like to see by myself. How the three-different theories produce identical numbers.

\section{Acknowledgement}

I would like to express the deepest appreciation to Professor El Syed Yehia El zyat, for fruitful discussion and scientific comments. In addition, a thank you to Professor Salah Arfa, who encourage me to complete this dissertation.

\section{References}

1. Farouk MA (2017) International journal of optics and applications. The wavy ray model, A new light model 7(1).

2. Farouk MA (2018) Research \& development in material science-Crimson Publisher's: How physics became a blind science. 2(5).

3. Farouk MA (2019) Evolution in mechanical engineering-Crimson Publishers. There Are No Photons 2(3).

4. From a life of physics covering lectures given at the International Center for Theoretical Physics, Trieste, Italy.

5. Understanding quantum physics-auser's manual michael morrison.

6. (2015) International journal of optics and applications, "The photon as a mass particle associated with a wave" Adnan Salih Al-Ethawi.

7. Biagio B, Giuseppe G (2016) Wave and photon descriptions of light historical highlights, epistemological aspects, and teaching practices. Eur J Physics 37(5): 055303.

8. Feynman RP, QED, The strange theory of light and matter.

9. Born M (1947) Atomic physics, Blackie and Son Limited, London and Glascow, UK.

10. DeBrolie L (1964) The current interpretation of wave mechanics-A critical study, Elsevier publishing company, Amsterdam, London, UK.

11. Longhurst RS (1973) Geometrical and physical optics, Longman Group Limited, London, UK.

12. Savelyev IV (1989) Physics, Mir Publishers, Moscow, Russia.

13. Meyer JR (1972) Introduction to classical and modern optics, Prenticehall, Inc., Engle wood cliffs, USA.

14. Schiff LI (1968) Quantum Mechanics, McGraw Hall, New York, UK.

15. Clark H (1973) A first course in quantum mechanics, London, UK.

16. Halliday D, Resnick R (1960) Physics for students of Science and Engineering, John Wiley and Sons Inc, USA.

17. Morrison MA (1990) Understanding quantum physics, Prentice-Hall International, USA.

18. Giancoli DC (1988) Physics for scientists and Engineers with modern physics, Prentice-Hall international, USA.

19. Feynman RP (1985) QED the strange theory of light and matter, Cox and Wymen Ltd.

20. Khanna, Gulati (1971) Fundamental of optics: geometrical and physical, Delhi, India.

21. Lowdin PO (1992) Some aspects of objectivity and reality in modern science, by reprinted from Foundations of Physics. 22(1). 
22. Taylor and Francis (2008) The nature of light-what is the photon, In: Chandrasekhar P, Kracklauer AF, Katherine C (eds.).

23. Salasnich L (2014) Quantum physics of light and matter a modern introduction to photonsm, Atoms and many body systems -(C) Springer International Publishing, Switzerland.
24. Constantine Meis, Light and vacuum, The wave-particle nature of light (C) 2015 World Scientific Publishing Co Pte Ltd, Singapore.

25. Haken H (1986) Light, Waves Atoms.

For possible submissions Click below: 